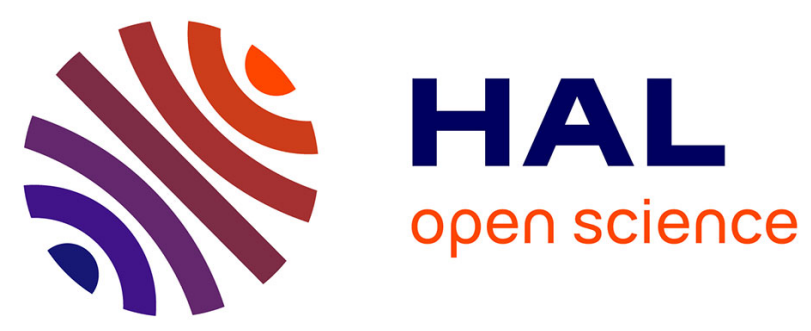

\title{
Eikonal Model Personalisation using Invasive Data to Predict Cardiac Resynchronisation Therapy Electrophysiological Response
}

Nicolas Cedilnik, Maxime Sermesant

\section{- To cite this version:}

Nicolas Cedilnik, Maxime Sermesant. Eikonal Model Personalisation using Invasive Data to Predict Cardiac Resynchronisation Therapy Electrophysiological Response. STACOM 2019 - 10th Workshop on Statistical Atlases and Computational Modelling of the Heart, Oct 2019, Shenzen, China. hal02368288

\section{HAL Id: hal-02368288 \\ https://hal.inria.fr/hal-02368288}

Submitted on 18 Nov 2019

HAL is a multi-disciplinary open access archive for the deposit and dissemination of scientific research documents, whether they are published or not. The documents may come from teaching and research institutions in France or abroad, or from public or private research centers.
L'archive ouverte pluridisciplinaire HAL, est destinée au dépôt et à la diffusion de documents scientifiques de niveau recherche, publiés ou non, émanant des établissements d'enseignement et de recherche français ou étrangers, des laboratoires publics ou privés. 


\title{
Eikonal Model Personalisation using Invasive Data to Predict Cardiac Resynchronisation Therapy Electrophysiological Response
}

\author{
Nicolas Cedilnik ${ }^{1,2,3}$ and Maxime Sermesant ${ }^{1,2,3}$ \\ 1 Université Côte d'Azur, France \\ 2 Inria Sophia Antipolis - Mediterranée Research Centre, France \\ 3 IHU Liryc, Bordeaux, France \\ nicolas.cedilnik@inria.fr
}

\begin{abstract}
In this manuscript, we personalise an Eikonal model of cardiac wave front propagation using data acquired during an invasive electrophysiological study. To this end, we use a genetic algorithm to determine the parameters that provide the best fit between simulated and recorded activation maps during sinus rhythm. We propose a way to parameterise the Eikonal simulations that take into account the Purkinje network and the septomarginal trabecula influences while keeping the computational cost low. We then re-use these parameters to predict the cardiac resynchronisation therapy electrophysiological response by adapting the simulation initialisation to the pacing locations. We experiment different divisions of the myocardium on which the propagation velocities have to be optimised. We conclude that separating both ventricles and both endocardia seems to provide a reasonable personalisation framework in terms of accuracy and predictive power.
\end{abstract}

Keywords: electrophysiology, computer model, personalisation, cardiac resynchronisation therapy

\section{Introduction}

For our participation in the STACOM piggyCRT challenge, we decided to use the Eikonal model of cardiac electrophysiology (EP). Using the fast marching method, simulations using this model are very fast to solve, which makes them both particularly suited to a clinical workflow [1] and easy to personalise. Moreover, as we are only interested in local activation times, the Eikonal model is relevant.

We determined the optimal parameters for this model, i.e., the parameters that minimise the discrepancy between the recorded and the simulated pre-cardiac resynchronisation therapy (CRT) activation maps, for each pig. This model personalisation was then used to predict the post-CRT activation maps using the same parameters (except for the initialisation of the propagation). 


\section{Model personalisation: general framework}

\section{$2.1 \quad$ Eikonal model}

The Eikonal model of cardiac electrophysiology outputs an activation map, i.e., local activation times (LATs) and is defined as follows:

$$
v \sqrt{\nabla T T^{t} D \nabla T}=1
$$

where $T$ is the local activation time, $v$ is the local conduction velocity and $D$ the anisotropic tensor to account for the fibre orientation. We experimented both with fibre orientations generated using the classic Streeter model and the provided OTRBM model.

To make it possible to use multiple onset locations with different delays, we ran one simulation $T_{i}$ for each onset $i$. We then added the desired onset delay $d_{i}$ to the whole activation map and combined them into a final activation map by choosing the minimal LAT for each element $X$ of the domain $\Omega$ :

$$
T_{\text {final }}=\min _{\forall X \in \Omega}\left(T_{1}(X)+d_{1}, T_{2}(X)+d_{2}, \ldots\right)
$$

Instead of solving the equation on the unstructured grid provided by the challenge, we decided to voxelise them, i.e., to define the domain on a regular lattice of 1 cubic millimetre resolution. Two reasons motivated this choice:

- morphological information on an individual heart is generally obtained from the segmentation of imaging data, which is naturally of this form,

- the fast marching method is faster on Cartesian grids.

As for the implementation, we used open-source fast marching routines available online [2].

\subsection{Parameter fitting with CMA-ES}

We used the covariance matrix adaptation - evolution strategy (CMA-ES) [3] genetic algorithm to fit our model parameters to the recorded EP maps. This approach has been used before for a similar challenge [4], and is well suited for multi-parameters, non-convex optimisation problems.

We chose to minimise the root median square difference between the recorded data and the simulation output. This choice is justified by the noise on the training data probably due to the acquisition itself and to its registration on the image-derived myocardial geometry. This could lead to outliers driving the root mean square error. 


\section{$3 \quad$ Velocities and domain division}

Given this framework, the main parameter that we tried to personalise was the local conduction velocity. But what is the optimal domain decomposition to define the number of local parameters to estimate?

Using different velocities for each voxel would both be impractical (too many parameters to optimise) and does not make sense from a physiological standpoint. Moreover, it would probably result in massive over-fitting to the pre-CRT maps with lower predictive power.

Keeping this in mind, we first tried to optimise a global speed for the whole domain, but also tried by individualising:

- both endocardia to capture both the Purkinje network (PN) and the left bundle branch block influences,

- both ventricle walls, for the same reason,

- the septum, for the same reason and because propagation through the septum could be much slower due to fibre orientation,

- the scar if present,

- the 17 AHA segments of the left ventricle (LV), to determine if this would be beneficial for the personalisation.

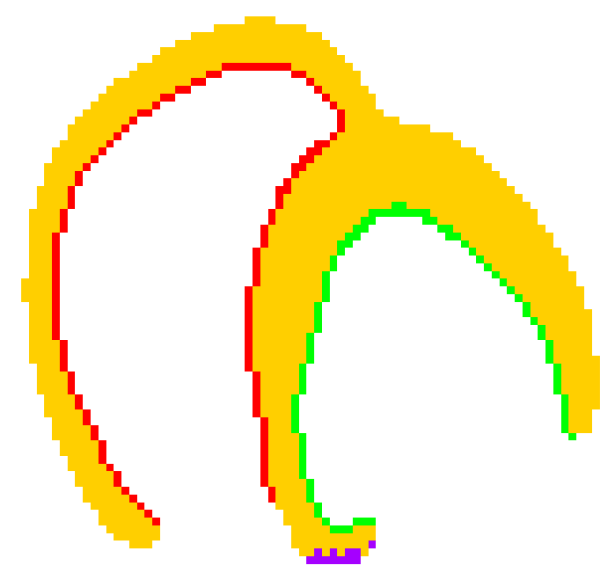

Figure 1: An example of domain division. yellow: wall, red: RV endocardium, green: RV endocardium, purple: connective tissue (outside the domain)

As the optimisation process is reasonably fast with our framework, we decided to test several combinations of these "velocity zones", as shown in Figure 5. 


\section{Onsets}

Besides the local propagation velocity, the Eikonal model requires to specify starting points for the wave front propagation. Choosing such points for the pre-CRT sinus rhythm maps is not trivial at all.

\subsection{Locations}

Ideally, the simulated pre-CRT maps should use the atrio-ventricular (AV) node as unique onset. We first tried to parameterise our model in such a way, but this approach rapidly proved very inefficient due to the massive influence of the septomarginal trabecula (ST) in the activation of the right ventricle (RV). As a consequence, it seemed more reasonable to use two different onsets, both in the RV endocardial layer. Unfortunately, the pre-CRT maps did not include any EP study of the RV endocardial surface.

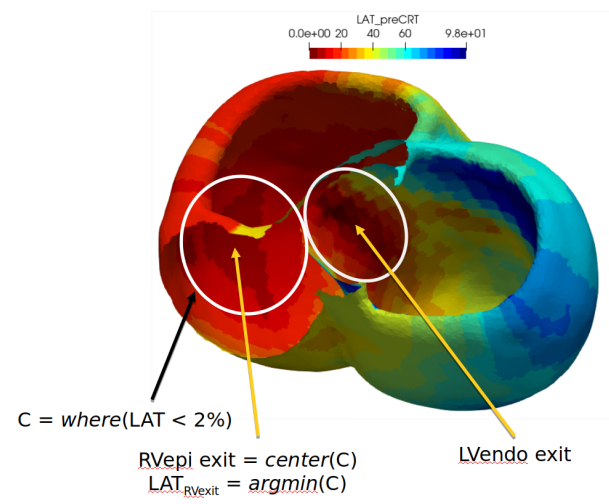

Figure 2: Determination of onset locations for pre-CRT maps

To overcome this limitation of the personalisation data, we chose the centre of gravity of all the points whose pre-CRT LATs were below the second percentile of a given area and picked the closest RV endocardium point. Picking up the point with the smallest LAT may sound more relevant, but because of the propagation spread, likely due to the PN, the simulations fit better using the "percentile" way (more on this in subsection 4.3) This was done for both the LV area, approximately locating the LV onset and the RV area, approximately locating the ST epicardial exit point, as illustrated in Figure 2. 


\subsection{Delays}

To determine the delays associated to these onsets (see Equation 2) we proceeded as follows:

1. Run an Eikonal simulation (Equation 1) for each onset location.

2. Choose the delay such that the lowest LAT of the simulation match the data, respectively for the $\mathrm{LV}$ endocardium (LV onset) and the RV epicardium (ST epicardial breakthrough).

\subsection{Radii}

Picking unique points for the onsets caused the optimisation to converge on unrealistically fast velocities to compensate for the spreading of the early activation due to the PN. It seemed logical to overcome this difficulty by "dilating" our onsets. To chose the radius of these dilations, we conducted the following study:

1. We fixed the endocardial velocity as $3 \mathrm{~m} / \mathrm{s}$ and scar velocity at $0.1 \mathrm{~m} / \mathrm{s}$.

2. We experimented a wide array of velocities for the rest of the domain, between 0.5 and $4 \mathrm{~m} / \mathrm{s}$.

3. For each velocity, we tested different onset radii, between 0 and $30 \mathrm{~mm}$.

4. We looked for the optimal myocardial velocity/onset radius combination, i.e., to combination that minimised the median square root error between the EP data and simulations.

The results of this study are shown on Figure 3 .
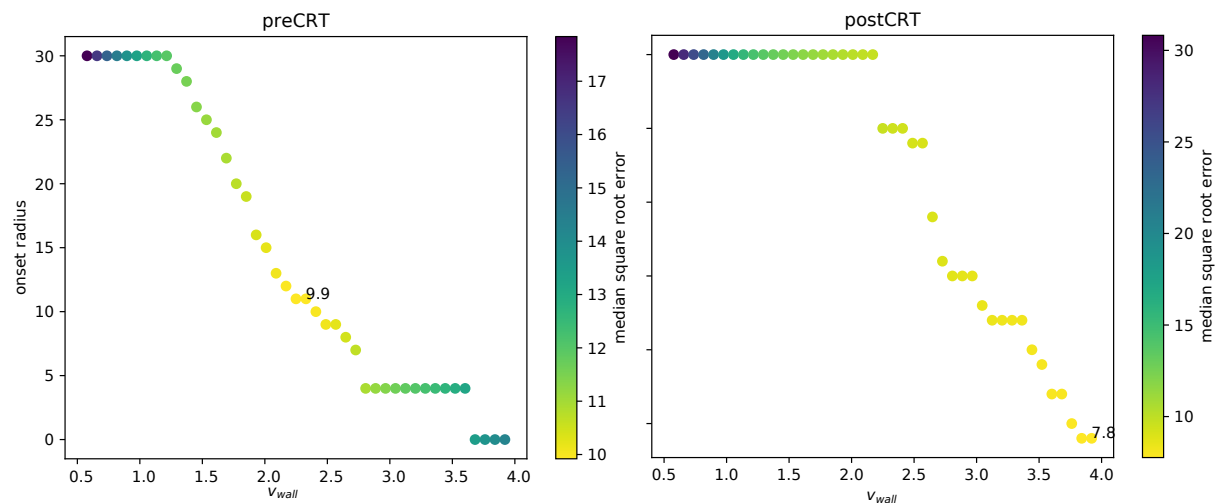

Figure 3: Combinations of onset radius and wall speed that result in the best match between simulation and EP data. Endocardial speed was here set to 3 $\mathrm{m} / \mathrm{s}$, scar speed to $0.1 \mathrm{~m} / \mathrm{s}$. 
Empirically, we could determine that an onset radius of $10 \mathrm{~mm}$ for the preCRT simulations and $2 \mathrm{~mm}$ for the post-CRT simulations allowed physiologycompatible velocities.

\section{Constraints}

We had to define parameter bounds for the optimisation process. We experimented with 3 types of regional constraints:

1. "Physiological": $v_{\text {scar }} \in[0,0.5], v_{\text {wall }} \in[0.5,1], v_{\text {endo }} \in[1,4]$

2. "Loose": $v_{\text {scar }} \in[0,1], v_{\text {wall }} \in[0.5,2], v_{\text {endo }} \in[1,6]$, to take into account the fact that the scar might be coarsely located

3. "No constraints": $v \in[0.1,4]$

To add a confidence estimation and to evaluate to the relevance of the fibre orientation, the anisotropy ratio, defined as the ratio between the velocity in the transverse plane and the velocity in the fibre direction was also optimised: $r \in[0.2,1]$.

\section{Results}

An example of fitting and prediction is shown on Figure 4.

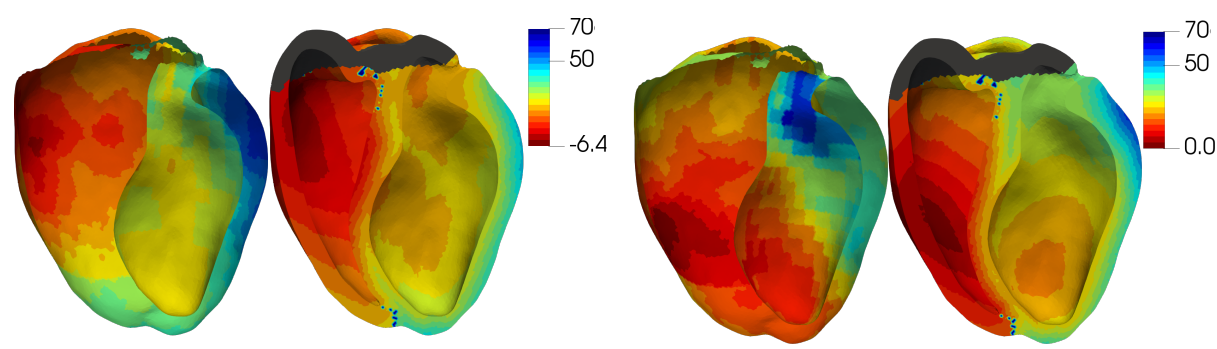

Figure 4: From left to right: recorded pre-CRT activation map, our model with fitted parameters, recorded post-CRT activation map, our model's prediction. Colours indicate LATs in ms.

\subsection{Performance}

The preCRT fit and CRT response prediction performances of the different constraints and domain divisions are shown in Figure 5. Pre-CRT fit ranged 


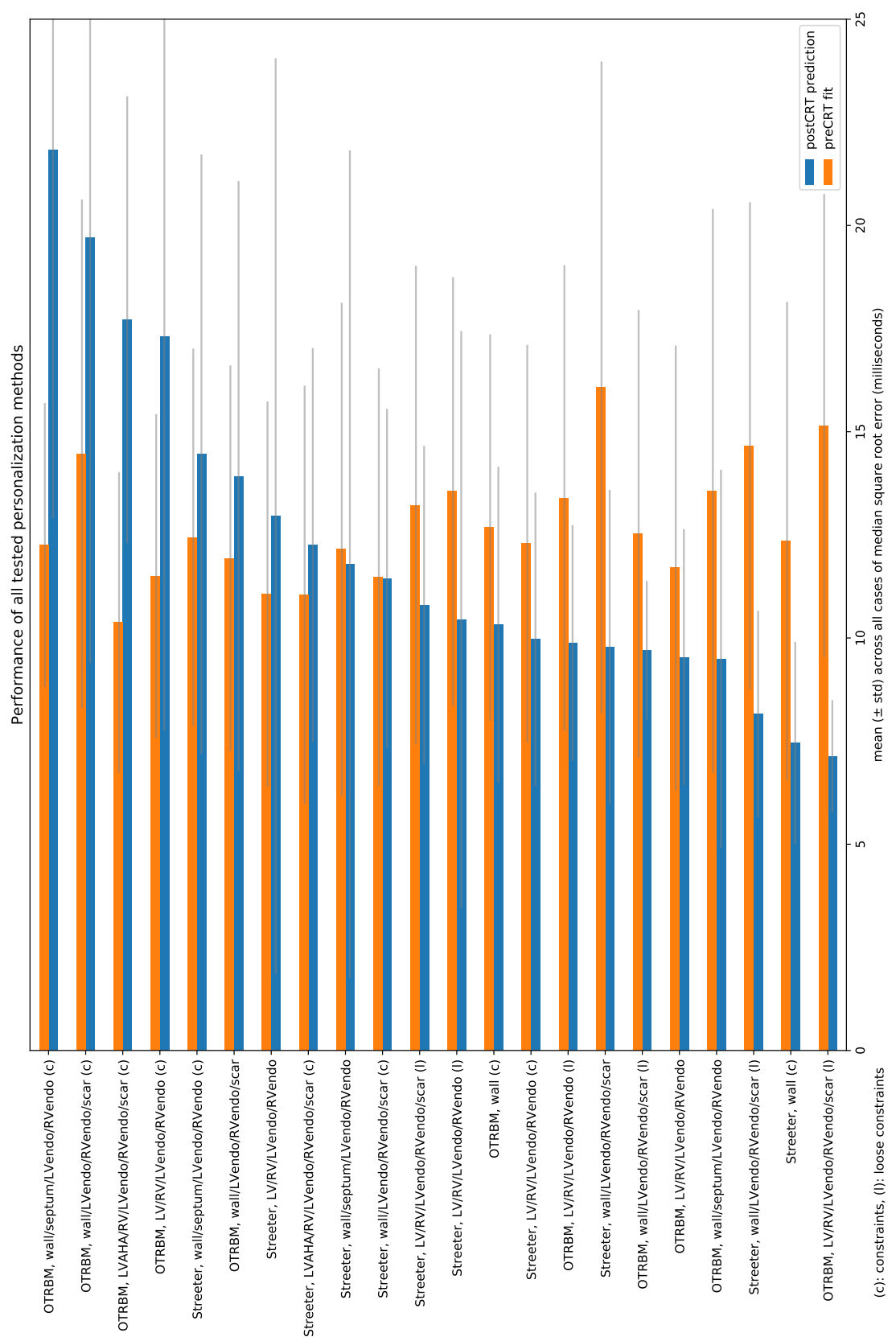

Figure 5: Fitting and prediction performance of the different domain divisions we experimented 
from 9 to $17 \mathrm{~ms}$ of median square root difference, while post-CRT prediction performance ranged from 7 to $22 \mathrm{~ms}$.

As expected, a very good preCRT fit is not correlated with a better postCRT prediction, but seems to rather be a sign of over-fitting.

The best approach in terms of prediction performance seems to be using OTRBM fibres and different speeds for both ventricles and both endocardia.

\subsection{Parameter fitting}

Taking a closer look at the optimal parameters, we realised that "physiological" constraints were too strict: best parameters were virtually always $4 \mathrm{~m} / \mathrm{s}$ for both endocardia and $1 \mathrm{~m} / \mathrm{s}$ for both walls.

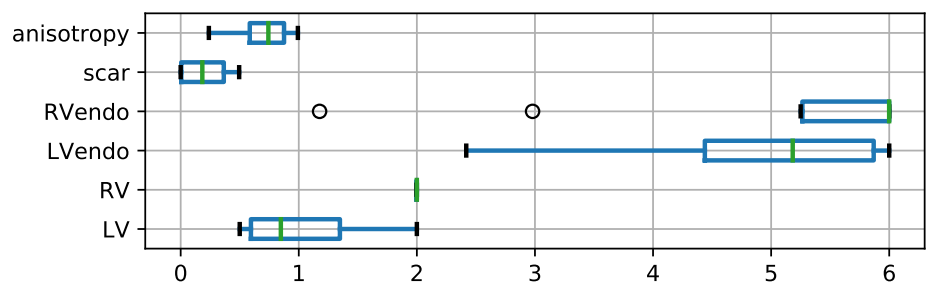

Figure 6: Distribution of parameters obtained with the "best" domain division and constraints combination.

In these conditions, personalisation did not seem to be really interesting and this is what motivated our experiments with "loose" constraints. As can be seen in Figure 6 and 7, this approach made proper personalisation possible.

\section{Discussion}

As we were given a very small dataset (3 post-CRT maps) to evaluate the prediction performance, it is really difficult to draw conclusions as to which approach really provides the best personalisation. However it seems clear that dividing the domain in small zones, e.g. the LV AHA segments is both detrimental to the prediction performance and the personalisation duration. We lacked time to explore other parameters combination, for instance, different anisotropy ratios by domain division or even looser constraints.

Our main contribution probably lies in the way we defined the onsets for the pre-CRT simulations and the fast framework proposed. In a clinical setting, 

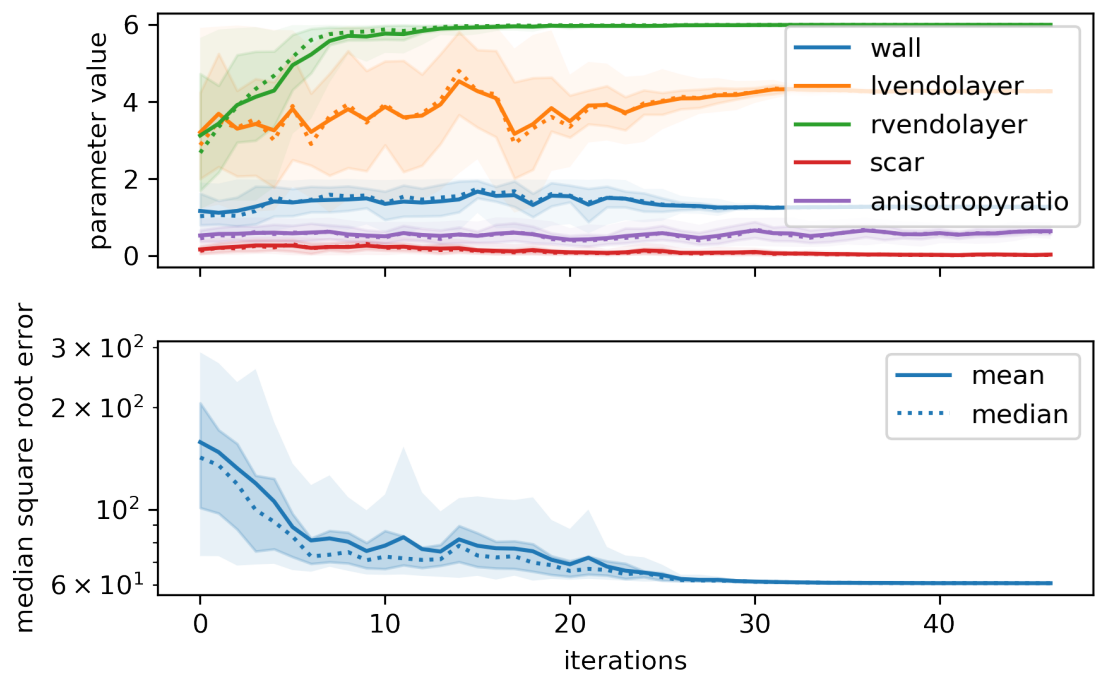

Figure 7: Evolution of the parameters' values (top) and loss function (bottom) using "loose" constraints for the pig "lali19" during the optimization process. The coloured surfaces represent the [5-95] (light) and [25-75] (darker) percentiles of the parameters (resp. the loss).

personalisation could probably be enhanced with imaging data $[5,6]$ and possibly ECGI data, and CRT response has to be evaluated with mechanical simulations [7].

\section{References}

1. Cedilnik, N., Duchateau, J., Dubois, R., Sacher, F., Jaïs, P., Cochet, H., Sermesant, M.: Fast Personalized Electrophysiological Models from CT Images for Ventricular Tachycardia Ablation Planning. EP-Europace. 20, (2018).

2. Mirebeau, J.-M.: Riemannian fast-marching on cartesian grids using Voronoi's first reduction of quadratic forms, https://hal.archives-ouvertes.fr/hal-01507334, (2017).

3. Hansen, N., Akimoto, Y., Baudis, P.: CMA-ES/pycma on Github, https://doi.org/ 10.5281/zenodo.2559634, (2019).

4. Giffard-Roisin, S., Fovargue, L., Webb, J., Molléro, R., Lee, J., Delingette, H., Ayache, N., Razavi, R., Sermesant, M.: Estimation of Purkinje Activation from ECG: An Intermittent Left Bundle Branch Block Study. Statistical Atlases and Computational Models of the Heart. Imaging and Modelling Challenges. pp. 135-142. Springer, Cham (2016).

5. Camara, O., Sermesant, M., Lamata, P., Wang, L., Pop, M., Relan, J., Craene, M. de, Delingette, H., Liu, H., Niederer, S., Pashaei, A., Plank, G., Romero, D., Sebastian, R., Wong, K.C.L., Zhang, H., Ayache, N., Frangi, A., Shi, P., Smith, N., Wright, G.: Inter-Model Consistency and Complementarity: Learning from ex-vivo Imaging and Electrophysiological Data towards an Integrated Understanding of Cardiac Physiology. (2011). 
6. Chen, Z., Cabrera-Lozoya, R., Relan, J., Sohal, M., Shetty, A., Karim, R., Delingette, H., Gill, J., Rhode, K., Ayache, N., others: Biophysical Modeling Predicts Ventricular Tachycardia Inducibility and Circuit Morphology: A Combined Clinical Validation and Computer Modeling Approach. Journal of cardiovascular electrophysiology. 27, 851-860 (2016).

7. Sermesant, M., Chabiniok, R., Chinchapatnam, P., Mansi, T., Billet, F., Moireau, P., Peyrat, J.-M., Wong, K.C.L., Relan, J., Rhode, K., Ginks, M., Lambiase, P., Delingette, H., Sorine, M., Rinaldi, C.A., Chapelle, D., Razavi, R., Ayache, N.: Patient-Specific Electromechanical Models of the Heart for Prediction of the Acute Effects of Pacing in CRT: A First Validation. (2012). 